\title{
Imaging Informatics: 25 Years of Progress
}

\author{
J. P. Agrawal', B. J. Erickson' ', C. E. Kahn, Jr. ${ }^{2}$ \\ Department of Radiology, Mayo Clinic, Rochester, MN, USA \\ 2 Department of Radiology and Institute for Biomedical Informatics, University of Pennsylvania, \\ Philadelphia, PA, USA
}

\begin{abstract}
Summary
The science and applications of informatics in medical imaging have advanced dramatically in the past 25 years. This article provides a selective overview of key developments in medical imaging informatics. Advances in standards and technologies for compression and transmission of digital images have enabled Picture Archiving and Communications Systems (PACS) and teleradiology. Research in speech recognition, structured reporting, ontologies, and natural language processing has improved the ability to generate and analyze the reports of imaging procedures. Informatics has provided tools to address workflow and ergonomic issues engendered by the growing volume of medical image information. Research in computeraided detection and diagnosis of abnormalities in medical images has opened new avenues to improve patient care. The growing number of medical-imaging examinations and their large volumes of information create a natural platform for "big data" analytics, particularly when joined with high-dimensional genomic data. Radiogenomics investigates relationships between a disease's genetic and gene-expression characteristics and its imaging phenotype; this emerging field promises to help us better understand disease biology, prognosis, and treatment options. The next 25 years offer remarkable opportunities for informatics and medical imaging together to lead to further advances in both disciplines and to improve health.
\end{abstract}

\section{Keywords}

Diagnostic imaging; radiology information systems; decision support systems, clinical; image interpretation, computerassisted; medical informatics

Yearb Med Inform 2016;Suppl 1:S23-31 hitp:://dx.doi.org/10.15265//YS-2016-s004 Published online June 30, 2016

\section{Introduction}

Medical imaging encompasses radiology, cardiology, pathology, endoscopy, and all of the other clinical specialties in which the interpretation and use of images applies to a patient's health [1]. The scientific discipline of medical imaging informatics - which seeks to manage and apply biomedical imaging information - has grown significantly in the past 25 years, as shown by the number of publications indexed each year in PubMed from 1990 through 2014 (Figure 1). The Journal of Digital Imaging, founded in 1987 and published by the Society for Imaging Informatics in Medicine (SIIM), formerly the Society for Computer Applications in Radiology, now publishes more than 100 articles each year. Since its founding in 2010, the Journal of Pathology Informatics has published more than 270 articles. Scientific advances in imaging informatics have found an audience in traditional specialty journals and in more general medical informatics journals. This article highlights some of the many advances in imaging informatics over the past 25 years.

\section{Structured Reporting, Ontologies, and Natural Language Processing}

Written communications between physicians form a crucial foundation of the medical record. Structured reporting is an approach to produce communications using controlled vocabulary and consistent, often template-based, report schemas. Point-andclick systems for structured reporting in radiology date back almost 40 years [2]. The
UltraSTAR system for ultrasound reporting provided a model for form-based data entry [3]. In the past 10-15 years, the widespread adoption of commercial speech-recognition systems has accelerated interest in template-based reporting. In radiology, the need to generate large numbers of reports requires a mechanism, such as a "talking template", that allows radiologists to keep their eyes on the images while dictating [4]. Structured reporting has been advocated to improve productivity, quality, and data capture $[5,6]$. Although work remains to be done [7], recent studies (e.g., [8]) have shown that structured reports generally have a positive impact on the reporting process. A notable approach, developed originally in oncologic imaging, is the Annotation and Image Mark-up (AIM) project, which provides a standardized, semantically interoperable information model for image-based annotations [9]. There also has been extensive effort to use controlled vocabularies and report templates in cardiology [10] and anatomic pathology [11].

An ontology is a knowledge model that humans can read and machines can process; it formally defines the terms in a domain of discourse and their relationships to one another. The National Center for Biomedical Ontology (NCBO) BioPortal site hosts more than 300 ontologies in a range of biomedical and health-related domains. Most general medical ontologies provide limited coverage of medical imaging. Over the past 10 years, the RadLex ontology ${ }^{1}$ has been developed to address that gap and provide a unifying lexicon for radiology. RadLex provides terminology for anatomy, diseases, imaging modalities, observations, and workflow; it includes subsets of relevant ontologies, such as the Foundational Model of Anatomy [12].

\footnotetext{
http://www.radlex.org/
} 
Agrawal et al.

The Radiology Gamuts Ontology defines a knowledge model for differential diagnosis in radiology [13]. An ontology of biomedical imaging has the potential to support the multi-scale understanding of disease by integrating radiological imaging and quantitative histopathology imaging with clinical and genomics data [14].

The clinical reports of most medical imaging procedures consist of narrative text. Imaging reports have served as materials for numerous investigations in natural language processing (NLP). In the 1990s, NLP research focused on parsing, regularization, and mapping of terms to a formal model to represent clinical information [15]. The development of negation-detection algorithms, such as NegEx [16], has afforded greater precision in identifying positive and negative assertions in narrative text. Work has also focused on extracting recommendations from imaging reports [17-19] and accurate detection of diseases and clinically relevant incidental findings [20,21].

\section{Standards and Interoperability}

"Digital Imaging and Communications in Medicine" (DICOM) and "Integrating the Healthcare Enterprise" (IHE) are pervasive in imaging informatics. DICOM is an international standard to communicate medical image information. IHE is a collaboration of professional societies and industry to improve the interoperability of healthcare information technology (IT) systems by defining preferred implementations of existing standards referred to as profiles, not creating new standards. Interoperability refers to the ability of different health care IT systems to communicate with one another and to interpret communicated information in the same way. The Health Level 7 (HL7) standard is used for communicating textual information between medical applications and devices and is beyond the scope of this review.

The year 1982 saw the first major meeting on Picture Archiving and Communication Systems (PACS), which was held in Newport Beach, CA. Many of the papers presented

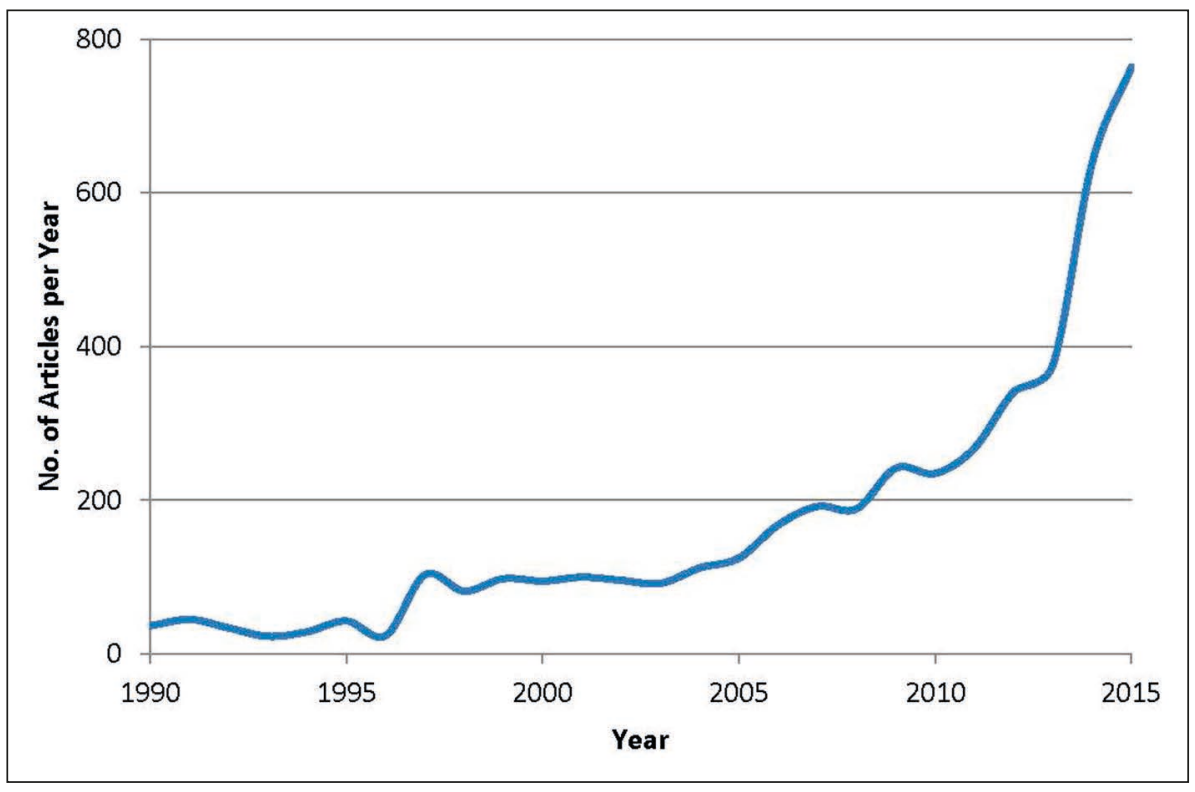

Fig. 1 Estimated number of articles per year in PubMed related to imaging informatics, using the query: (Radiology or Imaging or "Diagnostic Imaging") and (Informatics or "Image Processing" or "Image Analysis" or "Decision Support" or "Knowledge Representation" or PACS or "Radiology Information System").

echoed a common theme: digital imaging standards would be necessary if PACS were to be successfully implemented [22-25]. At the same time, radiologists and medical physicists using digital images for research began to express concern to the American College of Radiology (ACR) regarding the difficulty in accessing these images. In an effort to create a voluntary standard to address the problems in accessing digital images, the ACR and the National Electrical Manufacturers Association (NEMA) met in November 1983 to form the Digital Imaging and Communications Standards Committee [26]. DICOM began in 1985 as the ACR-NEMA 300-1985 Standard. The second version, ACR-NEMA 300-1988, was published in 1988. A number of vendors implemented this standard and a test of the implementations was performed in 1990 at Georgetown University [27]. Finalized in 1992, the third version of the standard transitioned to operation over standard computer networks and also changed its name to DICOM to be more inclusive of international participants [28]. Although DICOM started out as a radiology-specific standard, it has now extended into other fields such as cardiology, radiation therapy, endoscopy, pathology, ophthalmology, dentistry and oral surgery, and veterinary medicine [29].

DICOM is a standard for communicating and storing/archiving image information. The fundamental functional unit in DICOM is the Service-Object Pair (SOP). A SOP is the combination of a Service Class and an instantiated Information Object Definition (IOD). An IOD can be thought of as a form containing fields that are initially blank. Through the process of instantiation, the fields are assigned values, transforming the initially blank IOD into a DICOM Data Set. A Service Class refers to an application-level service, such as a request to store or transfer data. The SOP thus represents a command (Service Class) to be applied to the instantiated IOD (DICOM Data Set).

IHE is frequently misunderstood as a standard; it is not. It leverages existing standards to help integrate a diverse array of health care IT systems for the purpose of improving patient care [30]. It began in 1997 as a collaboration between the Radiological Society of North America (RSNA), the Healthcare Information and Management Systems Society (HIMSS), several academic centers, and a number of medical imaging vendors to solve integration problems 
encompassing the entire scope of healthcare informatics [31, 32]. Although IHE was initially a radiology-based endeavor, its scope has since expanded to anatomic pathology, cardiology, dentistry, endoscopy, eye care, IT infrastructure, laboratory, patient care coordination, patient care devices, pharmacy, quality, research and public health, and radiation oncology [33].

IHE utilizes use cases to model scenarios that not only describe workflows/processes comprising several systems/medical technologies but also require interoperability between the communicating components. A use case is a formal statement of a specific workflow, the inputs and outputs, and the actors that exchange transactions to accomplish a goal [34]. IHE uses existing standards (e.g., DICOM, HL7) to accomplish the goals of real-world use cases and provides implementation guidelines in the form of Integration Profiles. If existing standards are unable to successfully resolve a use case, IHE works with other standards committees to improve their standards. IHE also hosts global annual meetings, called Connectathons, where vendors gather to demonstrate IHE conformance and the interoperability of their products [33].

\section{Image Compression and Teleradiology}

Digital images are stored as a sequence of numbers with each number or set of numbers representing the brightness or color of a corresponding image pixel. The brightness or color of a pixel is termed the pixel value. The pixel of a digital grayscale image is represented by one (typically 16 bit) number while the pixel of a color image is represented by a set of numbers (if the Red-Green-Blue [RGB] model is used, three 8-bit numbers represent a single pixel). Pixel values are stored in order; the location of the pixel is implied by its location in the data set. In their native format, medical images contain redundancies; compression algorithms act to reduce or eliminate these redundancies to minimize storage size and transmission time. There are three main types of redundancies: coding redundancy; spatial, temporal, and bit-depth redundancy; and psychovisual redundancy. Although coding, spatial, temporal, and bit-depth redundancy reduction can be reversible or irreversible, psychovisual redundancy reduction can never be reversed. Spatial, temporal, and bit-depth redundancies are reduced by the process of transformation. Coding redundancy is reduced by the process of encoding. Psychovisual redundancy is (irreversibly) reduced by the process of quantization. Lossless compression algorithms perform transformation and encoding and are completely reversible; no image information is lost and the original image can be reconstituted exactly. Lossy compression algorithms perform transformation, quantization, and encoding and are irreversible; the reconstructed image is an approximation of the original image but not an exact replica. Although the Food and Drug Administration (FDA) finds the use of lossy compression acceptable, it requires labeling lossy compressed images as such when they are displayed.

Coding redundancy refers to the use of fixed-length coding to represent pixel values. Fixed-length coding uses the same number of bits to represent the value of each pixel. For typical medical grayscale images, pixels may have integer values between 0 and 4095 , meaning that each pixel has a size of 12 bits though in a 16-bit memory unit. While this representation may make sense intuitively, consider the fact that lower numbers such as 0 and 1 can be represented with only a single bit. Thus, when 0 or 1 is represented using fixed-length coding, 11 bits are unnecessarily used. Variable-length coding uses a variable number of bits to represent pixel values. There are two major methods of variable-length encoding to reduce coding redundancy: Huffman coding [35] and arithmetic coding [36]. Huffman coding constructs a binary tree of nodes using a bottom-up approach; pixel values with lowest frequency/probability are placed at the bottom of the tree and pixel values with highest frequency/probability are placed at the top of the tree. Left branches are assigned the value of 0 and right branches are assigned the value of 1 . Starting from the top of the binary tree, a pixel value is encoded as a string of the branch values leading to that pixel value. Decoding the pixel value follows a similar process: starting from the top of the binary tree, the string of encoded values is followed until a leaf of the tree is reached, which reveals the corresponding pixel value. Huffman coding assigns each individual pixel value a unique encoded value. Arithmetic coding takes a different approach to encoding pixel values. Instead of assigning each individual pixel value an encoded value, arithmetic coding assigns the entire image's set of pixel values one unique encoded real number value between 0 and 1 . Arithmetic coding assigns a range of real number values to each individual pixel value between 0 and 1 . The range for each pixel value is determined by its probability of appearing in the data set. Successive subdivision of the range between 0 and 1 into smaller intervals occurs until a final interval is reached that represents the encoded real number value. Decoding also performs successive subdivisions of the range between 0 and 1 , capturing the corresponding pixel values after each subdivision until the final pixel value is obtained. Although the algorithm for arithmetic coding is described using real numbers between 0 and 1, actual implementations use binary values and processes such as renormalization to avoid floating-point operations that lead to inaccuracies [37].

Spatial, temporal, and bit-depth redundancies occur because neighboring pixels and the same pixel in consecutive frames have similar or equal values [37]. The two main algorithms for reducing these types of redundancies are run-length encoding and the discrete cosine transform (DCT), both of which provide lossless compression. Run-length encoding utilizes a pair of values with one representing the number of consecutive pixels of the same value and the other representing the specific value. The DCT transforms the original image pixel values into more compact coefficients [38]. Both of these algorithms are used in the Joint Photographic Experts Group (JPEG) compression standard.

The JPEG compression standard includes both reversible and irreversible techniques [39]. Although not developed for medical imaging, JPEG compression has been adapted for radiological images [40]. JPEG compression begins by dividing the image 
into separate blocks of 8 pixels x 8 pixels. Pixel values are stored and then transformed using the DCT, resulting in $8 \times 8$ blocks of spectral coefficients. A small portion of the upper left corner of the DCT image contains the bulk of the information. Quantization is performed next and it is this step that makes JPEG a lossy compression algorithm. An $8 \times 8$ matrix called the quantization table contains the divisors that are used to divide the corresponding dividends contained in the $8 \times 8$ DCT spectral coefficient matrix. The quotients (quantized coefficients) are then rounded to the nearest integer. JPEG compression reorders the quantized coefficients in a zigzag pattern so that the largest values are placed first and long strings of zeros are placed at the end. This allows the use of an end-of-block symbol to indicate the rest of the $8 \times 8$ block of quantized coefficients is zero after the last nonzero coefficient is encoded [37].

The JPEG 2000 compression algorithm ${ }^{2}$ was designed to address the shortcomings of the standard JPEG compression standard and to use new image processing methods, such as wavelet compression, that allow for higher image quality at equal or higher compression ratios than those of standard JPEG and other compression methods. Among all of these capabilities, perhaps the most interesting is the ability to apply different compression ratios to different parts of an image. This would allow for specifying a lower compression ratio for the portion of the image containing the pathology of interest to retain the highest image quality and a higher compression ratio for the remainder of the image.

Assessing image quality after lossy compression is important. Although there are many metrics for evaluating image quality, the gold standard is visual evaluation of the lossy compression algorithm's impact on diagnostic quality. If the detection of true positive findings is not decreased and the detection of false positive findings is not increased, then the metrics are irrelevant. Finally, it is important to remember that storage costs continue to decrease over time. In the future, lossy compression may not be necessary for image storage; lossless compression alone may be sufficient. However,

\footnotetext{
http://www.jpeg.org/jpeg2000/
}

this statement has been true for decades but compression is still used because image sets grow larger at a rate similar to decreases in storage costs.

Teleradiology first began in the early 1990s with the advent of the Internet [41]. During this time, most Internet users made connections through standard analog phone lines. The relatively slow speed of dial-up connections made the transfer of diagnostic quality images without compression impractical. Consequently, compression algorithms were heavily utilized during this time to transfer images in a timely fashion. However, compression methods alone would not allow for the timely transfer of large data sets acquired through cross-sectional imaging. High-speed data transmission technology was needed to deal with the ever-increasing data size of imaging examinations. Once the technology for broadband Internet access became widespread, teleradiology began to expand and the need for compression algorithms decreased.

Teleradiology requires strong security measures to preserve patient confidentiality. A secure connection must be established between the imaging facility and the remote location where image interpretation will occur. Most teleradiology services use virtual private networks (VPNs) to establish secure connections between imaging facilities and remote interpreting locations. All data sent over a VPN is encrypted and decryption can only be accomplished through the use of an encryption key personalized to each authorized user. The VPN must also generate audit-logs to ensure that the system is not being misused.

Teleradiology has grown substantially over the last 25 years but what does the next 25 years hold? As more manned explorations into hostile environments, such as outer space, are undertaken, teleradiology will allow for the delivery of highly specialized imaging services in case of emergency [37]. The United States National Aeronautics and Space Administration (NASA) successfully pioneered the first extraterrestrial application of teleradiology by establishing a real-time link between experienced sonologists in Johnson Space Center, Houston, TX, who guided the crew aboard the International Space Station (ISS) in performing sono- graphic evaluation of shoulder integrity [42]. NASA also investigated the extraterrestrial performance of focused assessment with sonography for trauma (FAST) by establishing a real-time link between an ultrasound imaging expert in Mission Control Center, Houston, TX, who guided the crew aboard the ISS in performing the examination [43]. In a similar fashion, ground-based experts stationed in Mission Control Center, Houston, TX, guided the first extraterrestrial ultrasound examination of the genitourinary tract and the retroperitoneum in real time [44]. The positive outcomes from these projects prove that teleradiology has incredible potential both on Earth and beyond [45-47]. In the future, the remote delivery of image-guided therapeutic procedures using robotic assistance will become a reality.

\section{PACS and Imaging Workstations}

Vendors faced two main choices in designing PACS: whether to make the system "centralized" or "distributed." A centralized PACS stores images on a central server and needs high-speed transmission hardware to transmit the images to PACS workstations. A distributed PACS routes the images from the imaging device to the appropriate workstations based on rules reflecting typical workflow. For a distributed PACS system to be effective, the work must be predictable so that images can be sent from the imaging device to the correct workstations. Since the practice of medicine is often unpredictable, a distributed PACS architecture was difficult to implement successfully. With the development of large central servers and highspeed networks that can transmit images to workstations in a timely fashion, centralized PACS architecture has become the standard architecture and allows any workstation to retrieve any image stored in the PACS.

An important event that legitimized PACS as a feasible means to practice radiology was the Digital Imaging Network/ Picture Archiving and Communications System (DIN/PACS) contract, in which the Department of Defense released a request for 
proposal (RFP) for a PACS. In 1992, Fort Detrick saw the first installation of a PACS that utilized the ACR-NEMA 300-1988 standard. The Baltimore Veterans Administration Medical Center opened in 1993 as the first fully operational filmless facility in the United States [48].

An important component of any radiology reading room is the PACS workstation, which is essentially a very high-end computer. These workstations used to cost in the tens of thousands of dollars [49, 50]. A typical PACS workstation consists of two to four high-resolution liquid-crystal display (LCD) monitors. The graphics card used in a PACS workstation is responsible for providing connectivity to the displays and may also be responsible for performing much of the processing involved with zooming and panning images or in the rotation of a $3 \mathrm{D}$ rendering [51]. The ACR, American Association of Physicists in Medicine (AAPM), and SIIM have published jointly a technical standard of recommendations for the electronic practice of medical imaging. ${ }^{3}$ Workstations should have a graphic bit depth of at least 8 bits and LCD monitors with adequate brightness should be used. The ACR-AAPM-SIIM technical standard also provides specific workstation recommendations for parameters such as luminance, image presentation size, presentation support features, and pixel pitch and display size. PACS workstations should be able to communicate using the DICOM standard, although in most cases, they typically communicate with the central PACS server using an optimized proprietary protocol for routine image transfer. This approach is used because DICOM negotiations result in unnecessary overhead for routine transfers in a PACS.

The emergence of the Vendor Neutral Archive (VNA) is changing the original architecture of PACS and Radiology Information Systems (RIS). A VNA decouples the display aspect of PACS from the archiving layer; that is, imaging data is stored for long-term access in the VNA rather than in the PACS [52]. This decoupling eliminates the costly and time-consuming process of migrating

\footnotetext{
3 http://www.acr.org/ /media/ACR/

Documents/PGTS/standards/

ElectronicPracticeMedImg.pdf
}

data when a new PACS is installed. In the near future, PACS may be merged with RIS or may take over many of the traditional RIS functions. Since PACS and RIS frequently intercommunicate, merging the two would simplify the system architecture, would reduce the number of separate computers and systems, and should cost less and function more efficiently [53]. Still further in the future, PACS and RIS could conceivably be absorbed into the Electronic Medical Record (EMR), though this would be a complex and time-intensive endeavor.

\section{The "Image Explosion," the TRIP Initiative, and Ergonomics}

Medical imaging has seen the same explosion of data that has been experienced in other industries. In fact, the growth in volume of medical image data has demonstrated the exponential growth rates described by Moore's Law [54]. Technological advances allowing the rapid collection of image data and fast transfer of images have dramatically increased the number of images reviewed by radiologists. In the days before digital imaging, it was not uncommon for radiologists to review all of the previous day's CT or MR studies. Today, reviewing all of the previous day's studies is impossible; radiologists struggle to complete even the current day's workload. The Mayo Clinic in Jacksonville, FL, provides an excellent illustration of the profound expansion in the number of radiological images over time. In 1994, this institution acquired approximately 1,500 images daily; in 2002, an average of 16,000 images was acquired daily; by 2006, the number of images acquired daily exceeded 80,000 [55].

This "image overload" problem was recognized in the early 2000s and led to the creation of the Transforming the Radiological Interpretation Process (TRIP ${ }^{\mathrm{TM}}$ ) Initiative, which focused on developing strategies to deal with the medical image management crisis [55]. The TRIP ${ }^{T M}$ Initiative identified six research areas critical for managing the ever-increasing imaging volume: human per- ception, image processing and computer-aided detection, medical data visualization, image set navigation and usability, databases and systems integration, and methodology evaluation and performance validation [56]. Although technological and workflow solutions to help manage the large volume of data have been developed, continued work in medical image management is necessary to keep pace with the advancement of imaging technology.

Ergonomic factors are important to consider when designing a radiology reading room. In addition to maintaining a comfortable temperature, air flow, noise, and humidity level, the ACR, AAPM, and SIIM recommend that viewing conditions be optimized to minimize eye fatigue, that noise from computers and other devices be minimized, that proper chairs with lumbar support and adjustable height with armrests be made available, that workstation tables be height-adjustable, and that display devices be positioned an arm's length away from the viewer. To address work-related complaints from radiologists, researchers at Cornell University developed the Cornell Digital Reading Room Ergonomics Checklist in 2006 to evaluate the radiologist work environment and determine which areas need to be improved. ${ }^{4}$ The checklist is composed of six sections: display screens, input devices, workstation and workstation accessories, chair, ambient environment, and other factors. While working, radiologists are encouraged to keep their necks in a neutral position, to use good posture, and to change position often [57].

\section{Computer-aided Detection and Diagnosis}

Since the advent of computers for capturing and displaying radiological images, there has always been interest in determining whether computers may also be used to analyze images to detect and diagnose diseases [58]. Even the best radiologists make errors when interpreting images. The goal of

\footnotetext{
http://ergo.human.cornell.edu/ahprojects/ hronn06/HBthesisdefense.pdf
} 
computer-aided detection (CAD) and computer-aided diagnosis (CADx) is to minimize such errors from occurring and to improve overall accuracy and efficiency.

CAD and CADx have developed over the last 20 years. The first CAD system was developed by Nishikawa et al. in 1994 at the University of Chicago and was used to detect breast lesions and clustered microcalcifications on mammography [59]. In 1998, the first commercial CAD system, ImageChecker M1000 ${ }^{\circledR}$, developed by R2 Technology received FDA approval. CAD and CADx have also been applied to CT colonography and thoracic imaging. It is expected that the number of applications will continue to grow.

Although CAD and CADx systems can be developed using static program instructions, machine learning is a more novel algorithmic technique for making decisions dynamically. Machine learning evolved from the study of artificial intelligence. There are many approaches to developing a machine learning algorithm; however, they all use example cases to train the system. During the training process, the system learns how to assign appropriate weights to its inputs. Ultimately, the system develops the ability to make predictive decisions that are data-driven. Machine learning can be thought of as a way to transfer the knowledge of radiologists to the computers that will perform image analysis [60]. Adopting this approach to developing CAD and CADx provides the best results because the ideal system would function as an expert radiologist. Virtually all CAD and CADx systems utilize machine learning and perform four critical steps: preprocessing, segmentation, region of interest (ROI) analysis, and determination of whether detected structures represent true lesions. Early machine learning classification techniques, such as linear classifier, linear discriminant analysis (LDA), and simple neural networks have gradually evolved into more advanced techniques, such as support vector machines (SVMs), multiple-instance learning, and ensemble learning, which are in use today [60]. Growing literature suggests that when radiologists employ CAD and CADx for a second read, their diagnostic accuracy increases [61].

Image interpretation has three crucial components: detection, description, and differential diagnosis. The major focus of computer-aided applications for image analysis has traditionally been on detection. However, CADx is gaining more attention. CADx utilizing neural networks has been used in chest radiography to provide a differential diagnosis for ground glass opacities [62]. IBM's supercomputer Watson has also entered the fray as a new way to provide clinical decision support for the medical field. Watson's natural language processing capabilities and ability to mine enormous amounts of data in a short amount of time has the potential to revolutionize the way $\mathrm{CADx}$ would function in the near future.

As CAD and CADx algorithms become more sophisticated, the process of reviewing straightforward examinations will become increasingly automated. We fully expect that in the future, simple cases will only be occasionally reviewed by humans. Radiologists will be able to devote their time and attention to the more complex and difficult cases. This will lead to improved diagnostic accuracy and efficiency and, most importantly, to better patient outcomes.

\section{Three-dimensional Image Rendering}

Three-dimensional (3D) image rendering is used in multiple imaging modalities and often enhances the visualization of pathology. All 3D rendering techniques have four interdependent classes of $3 \mathrm{D}$ imaging operations: preprocessing (defining the object system to create a geometric model of the objects under investigation), visualization (viewing and comprehending the object system), manipulation (altering the objects [e.g., virtual surgery]), and analysis (quantifying information about the object system) [63]. The three main 3D rendering techniques used in medical imaging are shaded surface display (SSD; also known as surface rendering), maximum intensity projection (MIP), and $3 \mathrm{D}$ volume rendering [64].

SSD was the first $3 \mathrm{D}$ rendering technique applied to medical images [65]. Its development began in the 1970s and leveraged the computer graphics, image-processing techniques, data segmentation innovations, and display technology of the time [66-71]. In SSD, surfaces of structures of interest are determined within the volume of data and an image representing the derived surfaces is displayed [64]. The MIP 3D rendering technique was first applied to magnetic resonance (MR) angiography [65]. It remains a popular method for creating $3 \mathrm{D}$ computed tomography (CT) angiographic images [72-76]. As its name suggests, MIP preferentially displays higher intensity structures (e.g., contrast-enhanced vessels) at the expense of lower intensity structures; however, with minor modifications, MIP can be used to preferentially display lower intensity structures (minimum intensity projection) [65]. SSD and MIP typically make use of less than $10 \%$ of the available data [65]. In contrast, 3D volume rendering is capable of incorporating the entire data set [77-80]. 3D volume rendering sums the contributions of each voxel along a line extending from the viewer's eye through the entire data volume and then displays the resulting composite for each pixel [64]. Powerful computers are needed to perform 3D volume rendering in a timely fashion, since it is the most computationally-intensive technique.

As image-processing hardware continues to increase in speed and decrease in cost, the relatively low computational cost of SSD and MIP compared to volume rendering will become irrelevant; the selection of 3D rendering technique will only be determined by the specific clinical problem. Moreover, multiple different 3D rendering techniques may be performed for the same study because the information obtained is often complementary. 3D image rendering will continue to play an important role in helping radiologists interpret the ever-increasing volume of medical imaging data.

\section{Business Analytics and Operational Analysis}

Business analytics refers to the collection, analysis, and display of data that reflects activity within a business. In the case of a 
hospital or healthcare enterprise, this term refers not only to the standard business data of revenues and expenses, but also to how patients flow through a facility. How long are patients waiting to be seen in a given department or facility? How frequently does an expensive CT scanner sit idle? How often is it down for repair? How long has it been since its last preventive maintenance check? How many years have passed since it was purchased? Some of this data can reflect current issues-if no images have flown out of an imaging device for two hours, it could mean that there are no patients that need imaging; however, it could also mean that the device is down. Business analytics can help practice leaders sort out these questions and make good decisions.

In healthcare, there are few standards for business analytics. HL7 and DICOM are designed for conveying medical data, not business data. Billing systems do not address the immediate patient flow issues noted above. In response to this deficiency, SIIM in 2011 initiated SWIM-the SIIM Workflow Initiative in Medicine. ${ }^{5}$ SWIM began by identifying workflow steps that are frequently executed in an imaging department and contributed the lexicon of those workflow steps to RadLex. Once the lexicon of workflow is agreed upon, the next challenge is to collect and distribute the event information in a standard fashion [81]. There are currently a number of products that access workflow information using proprietary methods and then display the workflow information as a "dashboard" [82]. Although these tools are useful, the lack of standards means that such products are expensive to implement and are frequently disrupted when systems are upgraded or replaced. The proposed IHE profile called SOLE - the Standardized Operational Log of Events, ${ }^{6}$ seeks to address this challenge. This profile defines a standard way to collect events and how they may be accessed, leveraging the Audit Trail

\footnotetext{
5 http://siim.org/?page=swim_trip_ background

6 http://wiki.ihe.net/index. php?title=Standardized_Operational_ Log_of_Events_(SOLE)
}

and Node Authentication (ATNA) profile that is already in widespread use.?

Once standards are in place and in use for collecting and accessing workflow information, it becomes feasible to use workflow engine technology to assist the healthcare delivery process. Workflow engines (WFEs) are widely used in other industries, particularly manufacturing, where there are well-defined processes for a business. In healthcare, prior experiences with WFEs have been less positive because healthcare has many exception conditions leading to complex workflows [83, 84]. If those complexities are not included and modeled, the WFE will not be able to address the problems at hand and will actually put patients at risk rather than improve their health care. Imaging may be an area where WFEs can be implemented to great advantage. WFEs can improve efficiency and quality when adopted by components of an imaging department. Implementing WFEs in areas with few exceptions to workflow, and having a WFE that makes exception handling easy, is critical to long term success [85].

\section{9 "Big Data" and Radiogenomics}

"Big Data" in medical imaging encompasses the pixel data within imaging examinations as well as the interpretive report $[86,87]$. Sophisticated algorithms are necessary to mine and analyze Big Data due to its enormous size. Big Data analytics holds the potential to discover important relationships between a disease's imaging phenotype and its clinical characteristics [88-90]. As Big Data becomes more readily accessible in healthcare, providers have a duty to prevent its abuse by ensuring that Health Insurance Portability and Accountability Act (HIPAA) standards are followed and protected health information is secured.

Efforts over the past 25 years to sequence and understand the human genome have

\footnotetext{
7 http://wiki.ihe.net/index.php?title=Audit Trail_and_Node_Authentication
}

enabled "precision medicine," which seeks to improve clinical outcomes using detailed, personalized information to improve diagnosis, categorize disease, and guide treatment [91]. Imaging informatics plays a role in realizing the goals of precision medicine by making images and imaging-based information accessible and interoperable across clinical domains and biological scales [92]. The emerging field of radiogenomics investigates the relationships between a disease's genomic characteristics and its imaging phenotype to better understand the disease's biology, prognosis, and treatment options [93, 94]. Radiogenomics offers noninvasive tools to assess clinical and molecular characteristics of cancers, such as glioblastoma [95], renal cell carcinoma [96], and breast cancer [97], and to predict radiation therapy response [98]. As the field of radiogenomics continues to mature, it will play a crucial role in providing medicine that is tailored to the needs of each individual patient.

\section{Conclusion}

The past 25 years have seen remarkable advances in the science and applications of informatics in medical imaging. It is difficult to imagine what the next 25 years will bring, but already one can see some broad outlines. Teleradiology will remain a cost-effective healthcare service and will continue to expand. The vendor-neutral archive (VNA) separates the platforms for image viewing and for image storage - allowing both systems to innovate more rapidly - and can provide a single home for enterprise-wide healthcare image data. Efforts are underway to more completely integrate diagnostic services, such as radiology and pathology, to meet the needs of precision medicine. Advances in computer-aided interpretation of medical images may change the landscape of services that can be offered to patients. Finally, the collection and analysis of discrete clinical and operational information will provide a data-rich environment to drive evidence-based care and practice improvement. 


\section{References}

1. Bui AAT, Taira RK. Medical imaging informatics. New York: Springer; 2010.

2. Wheeler PS, Simborg DW, Gitlin JN. The Johns Hopkins radiology reporting system. Radiology 1976;119:315-9.

3. Bell DS, Greenes RA, Doubilet P. Form-based clinical input from a structured vocabulary: Initial application in ultrasound reporting. In: Frisse ME, editor. Proceedings of the 16th Annual Symposium on Computer Applications in Medical Care. New York: McGraw-Hill; 1992. p. 789-90.

4. Sistrom CL. Conceptual approach for the design of radiology reporting interfaces: the talking template. J Digit Imaging 2005;18:176-87.

5. Sistrom CL, Langlotz CP. A framework for improving radiology reporting. J Am Coll Radiol 2005;2:159-67.

6. Dunnick NR, Langlotz CP. The radiology report of the future: a summary of the 2007 Intersociety Conference. J Am Coll Radiol 2008;5:626-9.

7. Langlotz CP. Structured radiology reporting: are we there yet? Radiology 2009;253:23-5.

8. Marcovici PA, Taylor GA. Structured radiology reports are more complete and more effective than unstructured reports. AJR Am J Roentgenol 2014:203:1265-71.

9. Channin DS, Mongkolwat P, Kleper V, Sepukar $\mathrm{K}$, Rubin DL. The caBIG Annotation and Image Markup project. J Digit Imaging 2010;23:217-25.

10. Douglas PS, Hendel RC, Cummings JE, Dent JM, Hodgson JM, Hoffmann U, et al; American College of Cardiology Foundation (ACCF). ACCF/ACR/ AHA/ASE/ASNC/HRS/NASCI/RSNA/SAIP/ SCAI/SCCT/SCMR 2008 Health Policy Statement on Structured Reporting in Cardiovascular Imaging. J Am Coll Cardiol 2009;53:76-90.

11. de Baca ME, Madden JF, Kennedy M. Electronic pathology reporting: digitizing the College of American Pathologists cancer checklists. Arch Pathol Lab Med 2010;134:663-4.

12. Rubin DL. Creating and curating a terminology for radiology: ontology modeling and analysis. J Digit Imaging 2008;21:355-62.

13. Budovec JJ, Lam CA, Kahn CE, Jr. Radiology Gamuts Ontology: differential diagnosis for the Semantic Web. RadioGraphics 2014;34:254-64.

14. Smith B, Arabandi S, Brochhausen M, Calhoun M, Ciccarese P, Doyle S, et al. Biomedical imaging ontologies: A survey and proposal for future work. J Pathol Inform 2015;6:37.

15. Friedman $\mathrm{C}$, Alderson PO, Austin JH, Cimino JJ, Johnson SB. A general natural-language text processor for clinical radiology. J Am Med Inform Assoc 1994;1:161-74.

16. Chapman WW, Bridewell W, Hanbury P, Cooper GF, Buchanan BG. A simple algorithm for identifying negated findings and diseases in discharge summaries. J Biomed Inform 2001;34:301-10.

17. Lakhani P, Kim W, Langlotz CP. Automated extraction of critical test values and communications from unstructured radiology reports: an analysis of 9.3 million reports from 1990 to 2011. Radiology 2012;265:809-18.

18. Sistrom CL, Dreyer KJ, Dang PP, Weilburg JB,
Boland GW, Rosenthal DI, et al. Recommendations for additional imaging in radiology reports: multifactorial analysis of 5.9 million examinations. Radiology 2009;253:453-61.

19. Dang PA, Kalra MK, Blake MA, Schultz TJ, Stout M, Lemay PR, et al. Natural language processing using online analytic processing for assessing recommendations in radiology reports. J Am Coll Radiol 2008;5:197-204.

20. Pham AD, Neveol A, Lavergne T, Yasunaga D, Clément $\mathrm{O}$, Meyer $\mathrm{G}$, et al. Natural language processing of radiology reports for the detection of thromboembolic diseases and clinically relevant incidental findings. BMC Bioinform 2014;15:266.

21. Sohn S, Ye Z, Liu H, Chute CG, Kullo IJ. Identifying abdominal aortic aneurysm cases and controls using natural language processing of radiology reports. AMIA Jt Summits Transl Sci Proc 2013;2013:249-53.

22. Baxter B, Hitchner L, Maguire G, Jr. Characteristics of a Protocol for Exchanging Digital Image Information. 1st Intl Conf and Workshop on Picture Archiving and Communication Systems: SPIE Proceedings; 1982. p. 273-7.

23. Haney MJ, Johnston RL, O'Brien JWD. On standards for the storage of images and data. SPIE Medical Imaging1982. p. 294-7.

24. Schneider RH. The role of standards in the development of systems for communicating and archiving medical images. SPIE Medical Imaging 1982. p. 270-1.

25. Wendler T, Meyer-Ebrecht D. Proposed standard for variable format picture processing and a codec approach to match diverse imaging devices. SPIE Medical Imaging 1982. p. 298-307.

26. Horii SC. Introduction to "Minutes: NEMA Ad hoc Technical Committee and American College of Radiology's Subcommittee on Computer Standards". J Digit Imaging 2005;18:5-22.

27. Horii SC, Hill DG, Blume HR, Best DE, Thompson B, Fuscoe C, et al. An update on American College of Radiology-National Electrical Manufacturers Association standards activity. J Digit Imaging 1990;3:146-51

28. Bidgood WD, Jr, Horii SC. Introduction to the ACR-NEMA DICOM standard. RadioGraphics 1992;12:345-55

29. Kahn CE Jr, Carrino JA, Flynn MJ, Peck DJ, Horii SC. DICOM and radiology: past, present, and future. J Am Coll Radiol 2007;4:652-7.

30. Henderson M, Behlen FM, Parisot C, Siegel EL, Channin DS. Integrating the Healthcare Enterprise: a primer - part 4. The role of existing standards in IHE. RadioGraphics 2001;21:1597-603.

31. Channin DS. M:I-2 and IHE: Integrating the Healthcare Enterprise, year 2. RadioGraphics 2000;20:1261-2.

32. Channin DS, Parisot C, Wanchoo V, Leontiev A, Siegel EL. Integrating the Healthcare Enterprise: a primer - part 3. What does IHE do for ME? RadioGraphics 2001;21:1351-8.

33. IHE. Engaging HIT Stakeholders in a Proven Process. http://ihe.net/IHE_Process/. Accessed October 26, 2015.

34. Bittner K, Spence I. Use Case Modeling. Boston, MA: Addison-Wesley; 2002.

35. Huffman DA. A Method for the Construction of Minimum-Redundancy Codes. Proceedings of the
IRE 1952;40:1098-101

36. Abramson N. Information Theory and Coding. New York, NY: McGraw-Hill; 1963.

37. Kagadis GC, Langer SG. Informatics in Medical Imaging. Boca Raton, FL: Taylor \& Francis Group, LLC; 2012

38. Ahmed N, Natarajan T, Rao KR. Discrete cosine transform. IEEE Transactions on Computers 1974;C-23:90-3.

39. Wallace GK. The JPEG still picture compression standard. IEEE Transactions on Consumer Electronics 1992;38:xviii-xxxiv.

40. Gillespy T, 3rd, Rowberg AH. Displaying radiologic images on personal computers: image storage and compression - Part 2. J Digit Imaging 1994:7:1-12.

41. Hearaly BC, Viprakasit D, Johnston WK. The Future of Teleradiology in Medicine Is Here Today. In: Kumar S, Krupinski EA, editors. Teleradiology. Berlin, Heidelberg: Springer; 2008. p. 11-20.

42. Fincke EM, Padalka G, Lee D, van Holsbeeck M, Sargsyan AE, Hamilton DR, et al. Evaluation of shoulder integrity in space: first report of musculoskeletal US on the International Space Station. Radiology 2005;234:319-22.

43. Sargsyan AE, Hamilton DR, Jones JA, Melton S, Whitson PA, Kirkpatrick AW, et al. FAST at MACH 20: clinical ultrasound aboard the International Space Station. J Trauma 2005;58:35-9.

44. Jones JA, Sargsyan AE, Barr YR, Melton S, Hamilton DR, Dulchavsky SA, et al. Diagnostic ultrasound at MACH 20: retroperitoneal and pelvic imaging in space. Ultrasound Med Biol 2009:35:1059-67.

45. ESR white paper on teleradiology: an update from the teleradiology subgroup. Insights into imaging 2014:5:1-8

46. Ranschaert ER, Boland GW, Duerinckx AJ, Barneveld Binkhuysen FH. Comparison of European (ESR) and American (ACR) white papers on teleradiology: patient primacy is paramount. J Am Coll Radiol 2015;12:174-82.

47. Silva E, 3rd, Breslau J, Barr RM, Liebscher LA, Bohl M, Hoffman T, et al. ACR white paper on teleradiology practice: a report from the Task Force on Teleradiology Practice. J Am Coll Radiol 2013;10:575-85.

48. Siegel EL, Kolodner RM. Filmless Radiology. New York: Springer; 2001.

49. Dwyer SJ, Stewart BK. Clinical uses of grayscale workstations. In: Hendee WR, Trueblood JH, editors. 1993 AAPM Summer School on Digital Radiology. Madison, WI: Medical Physics Publishing; 1993. p. 241-64.

50. Stewart BK, Aberle DR, Boechat MI, et al. Clinical utilization of grayscale workstations. IEEE Eng Med Biol 1993;12:86-100.

51. Krupinski EA, Flynn MJ, Hirschorn DS. Displays IT Reference Guide for the Practicing Radiologist [serial online]. 2013. Available at: http:// www.acr.org/ /media/ACR/Documents/PDF/ Advocacy/IT\%20Reference\%20Guide/IT\%20 Ref\%20Guide\%20Displays.pdf. Accessed October 29, 2015.

52. Agarwal TK, Sanjeev. Vendor neutral archive in PACS. Indian J Radiol Imaging 2012;22:242-5.

53. Kagadis GC, Nagy P, Langer S, Flynn M, Starkschall G. Anniversary paper: roles of medical phys- 
icists and health care applications of informatics. Med Phys 2008;35:119-27.

54. Erickson BJ, Persons KR, Hangiandreou NJ, James EM, Hanna CJ, Gehring DG. Requirements for an enterprise digital image archive. J Digit Imaging 2001;14:72-82.

55. Andriole KP, Morin RL, Arenson RL, Carrino JA, Erickson BJ, Horii SC, et al. Addressing the coming radiology crisis--the Society for Computer Applications in Radiology transforming the radiological interpretation process (TRIP) initiative. J Digit Imaging 2004;17:235-43.

56. Andriole KP, Morin RL. Transforming medical imaging: the first SCAR TRIP conference. A position paper from the SCAR TRIP subcommittee of the SCAR research and development committee. J Digit Imaging 2006;19:6-16.

57. Harisinghani MG, Blake MA, Saksena M, Hahn $\mathrm{PF}$, Gervais D, Zalis M , et al. Importance and effects of altered workplace ergonomics in modern radiology suites. RadioGraphics 2004;24:615-27.

58. Lodwick GS, Turner AH Jr. Lusted LB, Templeton AW. Computer-aided analysis of radiographic images. J Chronic Dis 1966;19:485-96.

59. Nishikawa RM, Haldemann RC, Papaioannou J, et al. Initial experience with a prototype clinical intelligent mammography workstation for computer-aided diagnosis. Medical Imaging 1995: Image Processing. San Diego, CA: SPIE; 1995. p. 65-71.

60. Wang S, Summers RM. Machine learning and radiology. Med Image Anal 2012;16:933-51.

61. Chan HP, Doi K, Vyborny CJ, Schmidt RA, Metz $\mathrm{CE}$, Lam KL, et al. Improvement in radiologists' detection of clustered microcalcifications on mammograms. The potential of computer-aided diagnosis. Invest Radiol 1990; 25: 1102-10.

62. Ashizawa K, MacMahon H, Ishida T, Nakamura K, Vyborny CJ, Katsuragawa S, et al. Effect of an artificial neural network on radiologists' performance in the differential diagnosis of interstitial lung disease using chest radiographs. Am J Roentgenol 1999;172:1311-5.

63. Udupa JK. Three-dimensional visualization and analysis methodologies: A current perspective. RadioGraphics 1999;19:783-806.

64. Calhoun PS, Kuszyk BS, Heath DG, Carley JC, Fishman EK. Three-dimensional volume rendering of spiral CT data: theory and method. RadioGraphics 1999;19:745-64.

65. Heath DG, Soyer PA, Kuszyk BS, et al. Three-dimensional spiral CT during arterial portography: comparison of three rendering techniques. RadioGraphics 1995;15:1001-11.

66. Gouraud H. Continuous shading of curved surfaces. IEEE Transactions on Computers 1971;C20:623-9.

67. Phong BT. Illumination for computer generated pictures. Commun ACM 1975;18:311-7.

68. Robb RA, Greenleaf JF, Ritman EL, Johnson SA, Sjostrand JD, Herman GT al. Three-dimensional visualization of the intact thorax and contents: a technique for cross-sectional reconstruction from multiplanar x-ray views. Comput Biomed Res 1974;7:395-419.
69. Herman GT, Liu HK. Display of three-dimensional information in computed tomography. J Comput Assist Tomogr 1977;1:155-60.

70. Herman GT, Liu HK. Three-dimensional display of human organs from computed tomograms. Comput Graph Image Proc 1979;9:1-21.

71. Fuchs H, Kedem ZM, Uselton SP. Optimal surface reconstruction from planar contours. Commun ACM 1977;20:693-702.

72. Rubin GD, Dake MD, Napel SA, McDonnell CH, Jeffrey RB, Jr. Three-dimensional spiral CT angiography of the abdomen: initial clinical experience. Radiology 1993;186:147-52.

73. Castillo M. Diagnosis of disease of the common carotid artery bifurcation: CT angiography vs catheter angiography. AJR Am J Roentgenol 1993;161:395-8

74. Marks MP, Napel S, Jordan JE, Enzmann DR. Diagnosis of carotid artery disease: preliminary experience with maximum-intensity-projection spiral CT angiography. Am J Roentgenol 1993;160:1267-71.

75. Napel S, Marks MP, Rubin GD, Dake MD, McDonnell $\mathrm{CH}$, Song SM, et al. CT angiography with spiral CT and maximum intensity projection. Radiology 1992;185:607-10.

76. Brink JA, Lim JT, Wang G, Heiken JP, Deyoe LA, Vannier MW. Technical optimization of spiral CT for depiction of renal artery stenosis: in vitro analysis. Radiology 1995;194:157-63.

77. Drebin RA, Carpenter L, Hanrahan P. Volume rendering. SIGGRAPH Comput Graph 1988;22 65-74.

78. Ney DR, Fishman EK, Magid D, Drebin RA. Volumetric rendering of computed tomography data: principles and techniques. IEEE Comput Graph Appl 1990;10:24-32.

79. Fishman EK, Magid D, Ney DR, Chaney EL, Pizer SM, Rosenman JG, et al. Three-dimensional imaging. Radiology 1991;181:321-37.

80. Rubin GD, Beaulieu CF, Argiro V, Ringl H, Norbash AM, Feller JF, et al. Perspective volume rendering of CT and MR images: applications for endoscopic imaging. Radiology 1996;199:321-30.

81. Erickson BJ, Meenan C, Langer S. Standards for business analytics and departmental workflow. J Digit Imaging 2013;26:53-7.

82. Nagy PG, Warnock MJ, Daly M, Toland C, Meenan CD, Mezrich RS. Informatics in radiology: automated Web-based graphical dashboard for radiology operational business intelligence. RadioGraphics 2009;29:1897-906.

83. Huser V, Rasmussen LV, Oberg R, Starren JB. Implementation of workflow engine technology to deliver basic clinical decision support functionality. BMC Med Res Methodol 2011;11:43.

84. Mans R, van der Aalst W, Russell N. Implementation of a healthcare process in four different workflow systems. Eindhoven, Netherlands: Technische Universiteit Eindhoven; 2009.

85. Erickson BJ, Langer SG, Blezek DJ, Ryan WJ, French TL. DEWEY: the DICOM-enabled workflow engine system. J Digit Imaging 2014;27:309-13.
86. Kansagra AP, Yu JP, Chatterjee AR, Lenchik L, Chow DS, Prater AB, et al. Big Data and the future of radiology informatics. Acad Radiol 2016; 23 : $30-42$.

87. Pentecost MJ. Big data. J Am Coll Radiol 2015;12: 129.

88. Boubela RN, Kalcher K, Huf W, Nasel C, Moser E. Big Data approaches for the analysis of large-scale fMRI data using Apache Spark and GPU processing: a demonstration on resting-state fMRI data from the Human Connectome Project 2015:9:492.

89. Liebeskind DS, Albers GW, Crawford K, Derdeyn CP, George MS, Palesch YY et al. Imaging in StrokeNet: realizing the potential of Big Data. Stroke 2015;46:2000-6.

90. Margolies LR, Pandey G, Horowitz ER, Mendelson DS. BBreast imaging in the era of Big Data: structured reporting and data mining. AJR Am J Roentgenol 2016;206:259-64

91. Toward Precision Medicine: Building a Knowledge Network for Biomedical Research and a New Taxonomy of Disease. Washington (DC): National Academies Press; 2011.

92. Hsu W, Markey MK, Wang MD. Biomedical imaging informatics in the era of precision medicine: progress, challenges, and opportunities. J Am Med Inform Assoc 2013;20:1010-3.

93. Mazurowski MA. Radiogenomics: what it is and why it is important. J Am Coll Radiol 2015;12:862-6.

94. Rosenstein BS, West CM, Bentzen SM, et al. Radiogenomics: radiobiology enters the era of big data and team science. Int J Radiat Oncol Biol Phys 2014;89:709-13.

95. Gevaert O, Mitchell LA, Achrol AS, Xu J, Echegaray S, Steinberg GK, et al. Glioblastoma multiforme: exploratory radiogenomic analysis by using quantitative image features. Radiology 2014:273:168-74.

96. Karlo CA, Di Paolo PL, Chaim J, Hakimi AA, Ostrovnaya I, Russo P, et al. Radiogenomics of clear cell renal cell carcinoma: associations between CT imaging features and mutations. Radiology 2014;270:464-71.

97. Grimm LJ, Zhang J, Mazurowski MA. Computational approach to radiogenomics of breast cancer: Luminal A and luminal B molecular subtypes are associated with imaging features on routine breast MRI extracted using computer vision algorithms. J Magn Reson Imaging 2015;42:902-7.

98. Kerns SL, West CM, Andreassen CN, Barnett GC, Bentzen SM, Burnet NG, et al. Radiogenomics: the search for genetic predictors of radiotherapy response. Future Oncol 2014; 10: 2391-406.

\section{Correspondence to:}

Charles E. Kahn, Jr.

Department of Radiology

3400 Spruce Street, 1 Silverstein

Philadelphia, PA 19104

USA

E-mail: charles.kahn@uphs.upenn.edu 